\title{
Thoracoscopic assistance in tricuspid valvuloplasty in an unarrested state: a case report and literature review
}

\author{
Feng Zhao", Qingliang Chen", Zhigang Guo, Nan Jiang \\ Department of Cardiovascular Surgery, Tianjin Chest Hospital, Tianjin University, Tianjin, China \\ \#These authors contributed equally to this work. \\ Correspondence to: Zhigang Guo, MD; Nan Jiang, MD. Tianjin Chest Hospital, Tianjin University, No. 261, Taierzhuangnan Road, Jinnan District, \\ Tianjin 300222, China. Email: leftcure@163.com; jiangnantj@126.com.
}

\begin{abstract}
Tricuspid regurgitation, a common tricuspid lesion, consists of organic and functional tricuspid insufficiency (FTI). FTI is usually secondary to the valvular heart disease in left atrium. Pulmonary hypertension may result in right ventricular and tricuspid annular enlargement. This report documents our findings of tricuspid valve surgery under cardiac arrest with telescopic assistance. A 65-year-old female patient referred to our department received thoracoscope-associated tricuspid valvuloplasty. The patient exhibited a history of intermittent dyspnea and shortness of breath for 20 years, together with edema in the lower limbs for 3 months. A small incision was made, prior to an additional incision of about $3 \mathrm{~cm}$ in length before localization was performed at the lateral side of the 4th midclavicular line. The satellite hole was localized at the 5 th midaxillary line. The operation was completed under cardiopulmonary bypass with a beating heart. Echocardiogram (ECG) analysis 10 days post-surgery indicated no clinically significant findings. Finally, the patient was discharged with slight tricuspid regurgitation .Thoracoscopy-associated heart surgery reduces postoperative pain and shortens postoperative recovery time. It is in line with the concept of rapid recovery and beauty needs. Our data confirmed that thoracoscope-assisted tricuspid valvuloplasty in an unarrested state was effective for the treatment of patients with tricuspid insufficiency, secondary to post-cardiac surgery.
\end{abstract}

Keywords: Thoracoscope; tricuspid; valvuloplasty; unarrested; case report

Submitted Sep 07, 2020. Accepted for publication Dec 02, 2020.

doi: 10.21037/atm-20-6995

View this article at: http://dx.doi.org/10.21037/atm-20-6995

\section{Introduction}

Tricuspid regurgitation, a common tricuspid lesion, consists of organic and functional tricuspid insufficiency (FTI). The former commonly occurs in patients with rheumatic heart disease and rare patients with congenital heart diseases such as endocardial cushion defect and Ebstein anomaly. FTI is usually secondary to the valvular heart disease in left atrium. Pulmonary hypertension may result in right ventricular and tricuspid annular enlargement. Secondary FTI is mainly featured in adults with tricuspid disease (1). In clinical settings, patients with left valvular disease may present with FTI after cardiac valve surgery. Despite the improvements in mitral flow and pulmonary arterial pressure, the possibility of right ventricular and tricuspid annular enlargement still remains. The persistence of FTI may trigger severe right heart failure and death. FTI is therefore a major factor affecting the quality of life after left cardiac valve surgery. In a previous study, the prevalence of severe tricuspid insufficiency was $7-27 \%$ among patients in the long term after left cardiac valve surgery. In addition, it was usually associated with poor prognosis (2).

Patients presenting with FTI after left cardiac surgery may display long-term injury in the right heart, which is mainly featured by edema in the lower limbs and the whole body, together with chronic hepatic congestion, chronic renal insufficiency, pulmonary hypertension and poor systemic nutrition. Therefore, they show poor tolerance 


\section{Page 2 of 6}

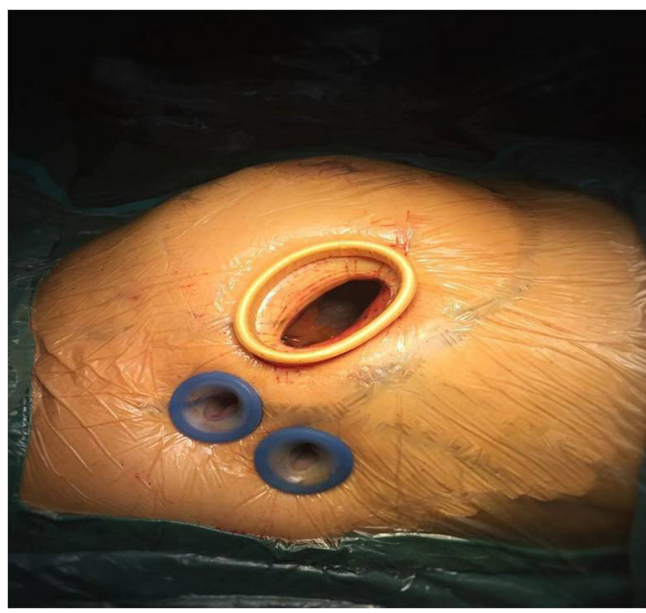

Figure 1 The telescope hole location.

to the surgery, with potentially many complications after conventional therapy. The classic procedure for tricuspid valve surgery is conventionally via thoracotomy, performed in the presence of cardiac arrest. For cases that received the second surgery, thoracotomy was required, which may involve extensive errhysis when separating the adhered pericardium, heart and large vessels. Mistakes during surgery may lead to structure damage, heart rupture, and hemorrhea, which is potentially lethal. Therefore, attenuation of surgical trauma and rapid post-operative recovery are crucial for patients who undergo tricuspid valve surgery. In this report, we present our findings from telescopic-assisted tricuspid valve surgery under cardiac arrest.

We present the following article in accordance with the CARE reporting checklist (available at http://dx.doi. org/10.21037/atm-20-6995).

\section{Case presentation}

All procedures performed in studies involving human participants were in accordance with the ethical standards of the institutional and/or national research committee(s) and with the Helsinki Declaration (as revised in 2013). Written informed consent was obtained from the patient.

A 65-year-old female patient was referred to our department exhibited a history of intermittent dyspnea and shortness of breath for 20 years together with edema in the lower limbs for 3 months.. The patient developed chest tightness, suffocation, palpitations, and inability to sleep supine at night before admission 20 years ago.

\section{Zhao et al. Thoracoscopic assistance in tricuspid valvuloplasty}

Ultrasonic echocardiogram (ECG) revealed rheumatic mitral stenosis (RMS). The patient subsequently underwent mechanical valve implantation, which later achieved obvious attenuation, and normal cardiac function. After discharge, she took warfarin and other drugs regularly, and the general condition was acceptable. More than 3 months before admission, the patient complained a progressive decline in daily activity tolerance, and there was no obvious cause for edema of both lower limbs and face, symmetrical pitting edema, accompanied by oliguria. Physical examination: symmetrical pitting edema of both lower limbs, jugular vein distended, Hepatic neck reflux sign $(+)$, heart rate 80 beats/min, absolute arrhythmia, unequal first heart sound, short pulse, audible mechanical valve opening sound in the mitral auscultation area, tricuspid A 4/6 grade systolic murmur can be heard in the valve auscultation area. The echocardiogram indicated: LA $56 \mathrm{~mm}$, RA $52 \mathrm{~mm}$, PAP $45 \mathrm{mmHg}$, EF 60\%, after mitral valve replacement, the function of the artificial valve was roughly normal, the tricuspid regurgitation was moderate to severe, pulmonary hypertension, and arrhythmia. The chest radiograph shows that the heart shadow is enlarged, and the heart-thoracic ratio is $70 \%$. ECG chart: ectopic heart rhythm, atrial fibrillation. The patient had a history of hypertension, breast cancer resection, and hysterectomy. The initial diagnosis was rheumatic heart disease, after mitral valve replacement, tricuspid regurgitation. After admission, she was given anticoagulation, cardiotonic, diuretic, ventricular rate control, and cardiac function stabilization treatments.

The patient received thoracoscope-associated tricuspid valvuloplasty 10 days after admission. Upon anesthesia and the establishment of extracorporeal circulation, a small incision was made prior to making an additional incision with a length of approximately $3 \mathrm{~cm}$ before localization at the lateral side of the 4th midclavicular line. The satellite hole was localized at the 5 th midaxillary line. The telescopic hole was localized at the anterior axillary line of the 4th intercostal space (Figure 1). Upon separation of the right atrium, enlargement in the tricuspid annulus was observed. After washing with saline, persistent repair was applied to the perforation hole near the posterior septum (Figure 2). Subsequently, the tricuspid annulus MC3 ring was inserted, prior to intermittent mattress suturing (Figure 3). The operation was completed under cardiopulmonary bypass with a beating heart. The operation time was 126 minutes, the cardiopulmonary bypass time was 78 minutes, and the tracheal intubation was removed 12 hours after the operation. The postoperative thoracic drainage volume 


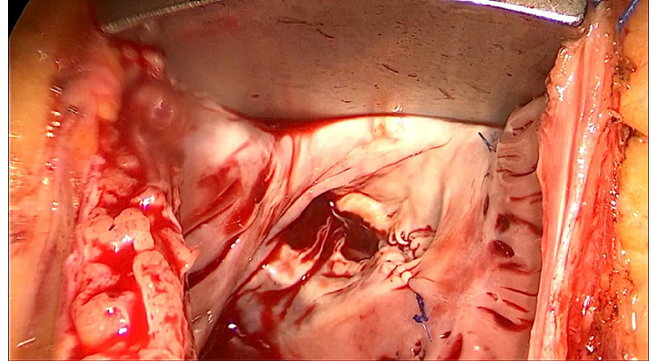

Figure 2 Persistent repair was given to the perforation hole near the posterior septum.

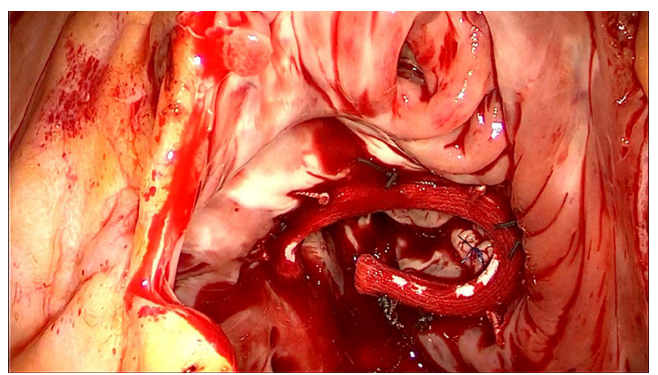

Figure 3 The tricuspid annulus MC3 ring was inserted, prior to the application of intermittent mattress suture.

was $50 \mathrm{~mL}$. No red blood cells were infused during or after the operation. After the drainage tube was removed, the patient could get out of bed. ECG analysis on day 10, post-surgery indicated no clinically significant findings. Finally, the patient was discharged with slight tricuspid regurgitation. We followed up the patient by telephone in the second year after the operation. The patient recovered well, with grade I cardiac function (NYHA), and no adverse complications such as atrioventricular block or stroke. The echocardiogram indicated a mild tricuspid regurgitation.

\section{Discussion}

Post-valve replacement FTI is a major challenge in cardiac surgery. However, limited focus has been directed to the tricuspid valve. According to our previous finding, FTI may be attenuated with the improvement of left heart function. Thus, no surgical treatment was applied during left cardiac valve surgery (3). An increasing number of studies have recently emerged, which indicated that most secondary FTIs would not attenuate with improvement of the left heart function. Furthermore this even led to failure of the right heart function $(4,5)$. In a previous study, Izumi et al. reported that approximately $12.9 \%$ of patients with no or mild tricuspid regurgitation may progress into severe tricuspid regurgitation during the 5-year follow-up period. A large proportion (23-37\%) of patients who underwent rheumatic mitral valve replacement 10 years go may present with severe tricuspid regurgitation (6). Dreyfus et al. reported that $34 \%$ of patients with mitral valve incompetence may develop tricuspid regurgitation approximately 5 years after mitral valvuloplasty (7). Moreover, patients with Ebstein anomaly and tetralogy of Fallot may develop severe tricuspid insufficiency after tricuspid valvuloplasty and cardiac surgery, respectively $(8,9)$.

FTI is often secondary to left heart valve disease. Because FTI is a functional rather than organic disease, there are fewer abnormalities in the valve leaflet structure. The main cause of tricuspid insufficiency is that severe left heart disease overloads the left atrium pressure. The reverse pressure load causes secondary pulmonary vein and arterial hypertension and pulmonary circulatory congestion. Eventually it causes enlargement and dysfunction of the right ventricle. The conformational change can further cause the expansion of the tricuspid annulus. The shape of the tricuspid annulus has changed from a saddle-like shape to an oblate shape, which makes the area of the tricuspid valve expand and the compliance of the annulus decreases. The valve leaflets and chordae can undergo displacement changes, which may affect the normal involution of the valve leaflets due to traction. On the other hand, FTI further causes right ventricular insufficiency and aggravates right ventricular remodeling. The increased diastolic pressure of the right ventricle causes pressure on the left ventricle, which will increase the left ventricular diastolic pressure and pulmonary circulation pressure. Eventually a vicious circle of mutual cause and effect is formed.

Patients with severe right heart insufficiency may present with severe decline in their quality of life with tricuspid insufficiency. To obtain satisfactory clinical efficiency, positive measures should be given in the presence of early stage right ventricular dysfunction. According to the guidelines proposed by American College of Cardiology/American Heart Association and European Society of Cardiology/European Association for Cardio-Thoracic Surgery, patients with moderate or severe tricuspid regurgitation, or enlargement in the tricuspid annulus of $>40 \mathrm{~mm}$ were recommended to undergo tricuspid surgery (I C). Patients who have previously received left cardiac valve surgery but not have severe pulmonary hypertension or obvious right ventricular systolic dysfunction, were recommended to undergo tricuspid repair 
or replacement again by ACC/AHA (type IIb C) or ESC/ EACTS (IIa C) $(10,11)$.

Currently, tricuspid valvuloplasty is the main form of treatment for tricuspid insufficiency (12) due to the hemodynamics of the venous system in the right heart. Tricuspid valve replacement may trigger high risks of thrombosis. Therefore, these patients may present a large risk for anti-coagulant therapy after surgery. In a previous study, the replacement efficiency of heart valve prosthesis for the tricuspid valve was inferior to that of the bicuspid valve and the aortic valve (13). Moreover, Marquis-Gravel et al. reported that the 10 -year survival among patients who received tricuspid valvuloplasty was higher than that of the counterparts that underwent tricuspid valve replacement (49\% vs. $38 \%, \mathrm{P}<0.05)$ (14). Therefore, restoration of the tricuspid valve function through valvuloplasty is well acknowledged.

The major methods for tricuspid insufficiency mainly include linear and prosthetic valve ring implantation. Extensive studies demonstrated that prosthetic valve ring implantation was crucial for FTI. For example, McCarthy et al. analyzed the recurrence of FTI within 8 years after the tricuspid valvuloplasty among 790 cases. The report indicated that the recurrent rate of FTI in the long-term was lower in patients who received tricuspid valvuloplasty, compared to those who underwent suturing plasty (15). Similarly, Tang et al. indicated that the survival and recurrent rate of FTI among the patients who underwent valve ring implantation compared those who received DeVega surgery (16). Moreover, Kara et al. suggested that tricuspid valve repair with an annuloplasty ring could improve the treatment outcome (17). Based on this finding, an Edwards MC3 ring was inserted to prevent the dilatation while maintaining the three-dimensional structure of the tricuspid valve ring, potentially preventing the recurrence of FTI.

The second tricuspid valve surgery is still currently a challenge due to surgical complexity and a high mortality rate, as well as postoperative complications. In many types of heart surgery, minimally invasive surgery assisted by thoracoscopy has been adopted (18). It is also suitable for high-risk patients with low ejection fraction and multivalvular disease $(19,20)$. Compared with the conventional therapy, the telescope-associated tricuspid valvuloplasty is featured by less trauma and pain, rapid recovery and clear vision for surgeon. Meanwhile, it prevented sternal dissection and attenuated the bleeding volume. In this study, cardiac arrest was performed where persistent perfusion of the oxygenated blood in the coronary arteries was observed. Our procedures could decrease the duration of myocardial ischemia and avoid ischemiareperfusion injury after aortic opening. After surgery, the patient required no blood transfusion and was able to walk after drainage tube removal. And the hospital stage was 1 week. The patient was followed up for at least 2 years, with no complications such as atrioventricular block or stroke observed.

In addition to traditional thoracotomy and thoracoscopic surgery, transcatheter tricuspid valve repair has also begun to be used in FTI treatment (21). The Sapien XT valve is placed in the superior vena cava and inferior vena cava, successfully reducing the system hemodynamic effects caused by severe tricuspid regurgitation (22). Trans-pericardial intrapericardial tricuspid annuloplasty (TRAIPTA) has been reported on a pig model (23). At present, a large number of studies have proved that for the surgical treatment of tricuspid valve insufficiency, tricuspid valve prosthetic ring is an effective and long-term effective method (24). TVP is currently the first choice for most FTI patients, and the midterm survival rate of patients can be significantly improved (25).

Tricuspid valvuloplasty in a unarrested state under the assistance of thoracoscope was effective for the treatment of tricuspid insufficiency, secondary to post-cardiac surgery. In addition, it could avoid the risk for conventional thoractomy and decrease the necessity for blood transfusion.

\section{Limitation}

This is the first case of thoracoscopic tricuspid valvuloplasty after mitral valve replacement in our department. There are no similar cases that can be used for controlled studies, so it is only a case report. This is also the shortcoming of this paper. We will continue to accumulate data on similar cases.

\section{Acknowledgments}

Funding: None.

\section{Footnote}

Reporting Checklist: The authors have completed the CARE reporting checklist. Available at http://dx.doi.org/10.21037/ atm-20-6995

Conflicts of Interest: All authors have completed the ICMJE uniform disclosure form (available at http://dx.doi. 
org/10.21037/atm-20-6995). ZG serves as an unpaid editorial board member of Annals of Translational Medicine from Aug 2020 to Jul 2021. The other authors have no conflicts of interest to declare.

Ethical Statement: The authors are accountable for all aspects of the work in ensuring that questions related to the accuracy or integrity of any part of the work are appropriately investigated and resolved. All procedures performed in studies involving human participants were in accordance with the ethical standards of the institutional and/or national research committee(s) and with the Helsinki Declaration (as revised in 2013). Written informed consent was obtained from the patient

Open Access Statement: This is an Open Access article distributed in accordance with the Creative Commons Attribution-NonCommercial-NoDerivs 4.0 International License (CC BY-NC-ND 4.0), which permits the noncommercial replication and distribution of the article with the strict proviso that no changes or edits are made and the original work is properly cited (including links to both the formal publication through the relevant DOI and the license). See: https://creativecommons.org/licenses/by-nc-nd/4.0/.

\section{References}

1. Antunes MJ, Rodriguez-Palomares J, Prendergast B, et al. Management of tricuspid valve regurgitation: Position statement of the European Society of Cardiology Working Groups of Cardiovascular Surgery and Valvular Heart Disease. Eur J Cardiothorac Surg 2017;52:1022-30.

2. Izumi C, Miyake $M$, Takahashi S, et al. Progression of isolated tricuspid regurgitation late after left-sided valve surgery. Clinical features and mechanisms. Circ J 2011;75:2902-7.

3. Braunwald NS, Ross J Jr, Morrow AG. Conservative management of tricuspid regurgitation in patients undergoing mitral valve replacement. Circulation 1967;35:I63-9.

4. Taramasso M, Vanermen H, Maisano F, et al. The growing clinical importance of secondary tricuspid regurgitation. J Am Coll Cardiol 2012;59:703-10.

5. Popal MS, Fu JT, Hu QM, et al. Intraoperative method based on tricuspid annular circumference in patients with mild or no tricuspid regurgitation during left-sided cardiac valve surgery for the prophylactic tricuspid annuloplasty. J Thorac Dis 2018;10:3670-8.
6. Izumi C, Iga K, Konishi T. Progression of isolated tricuspid regurgitation late after mitral valve surgery for rheumatic mitral valve disease. J Heart Valve Dis 2002;11:353-6.

7. Dreyfus GD, Corbi PJ, Chan KMJ, et al. Secondary tricuspid regurgitation or dilatation: which should be the criteria for surgical repair? Ann Thorac Surg 2005;79:127-32.

8. Said SM, Dearani JA, Burkhart HM, et al. Management of tricuspid regurgitation in congenital heart disease: is survival better with valve repair? J Thorac Cardiovasc Surg 2014;147:412-7.

9. Cheng JW, Russell H, Stewart RD, et al. The role of tricuspid valve surgery in the late management of tetralogy of fallot: collective review. World J Pediatr Congenit Heart Surg 2012;3:492-8.

10. Nishimura RA, Otto CM, Bonow RO, et al. 2014 AHA/ ACC guideline for the management of patients with valvular heart disease: a report of the American College of Cardiology/American Heart Association Task Force on Practice Guidelines. J Thorac Cardiovasc Surg 2014;148:e1-e132.

11. Vahanian A, Alfieri O, Andreotti F, et al. Guidelines on the management of valvular heart disease (version 2012): the Joint Task Force on the Management of Valvular Heart Disease of the European Society of Cardiology (ESC) and the European Association for Cardio-Thoracic Surgery (EACTS). Eur J Cardiothorac Surg 2012;42:S1-44.

12. Kilic A, Saha-Chaudhuri P, Rankin JS, et al. Trends and outcomes of tricuspid valve surgery in North America: an analysis of more than 50,000 patients from The Society of Thoracic Surgeons Database. Ann Thorac Surg 2013;96:1546-52.

13. Guenther T, Noebauer C, Mazzitelli D, et al. Tricuspid valve surgery: a thirty-year assessment of early and late outcome. Eur J Cardiothorac Surg 2008;34:402-9; discussion 409.

14. Marquis-Gravel G, Bouchard D, Perrault LP, et al. Retrospective cohort analysis of 926 tricuspid valve surgeries: clinical and hemodynamic outcomes with propensity score analysis. Am Heart J 2012;163:851-858.e1.

15. McCarthy PM, Bhudia SK, Rajeswaran J, et al. Tricuspid valve repair: durability and risk factors for failure. J Thorac Cardiovasc Surg 2004;127:674-85.

16. Tang GH, David TE, Singh SK, et al. Tricuspid valve repair with an annuloplasty ring results in improved longterm outcomes. Circulation 2006;114:I577-81.

17. Kara I, Koksal C, Cakalagaoglu C, et al. Recurrent 


\section{Page 6 of 6}

tricuspid insufficiency: is the surgical repair technique a risk factor? Tex Heart Inst J 2013;40:34-41.

18. Lan H, Cheng YG, Jia BC, et al. Clinical outcome of totally thoracoscopic cardiac surgery for mitral valve replacement: a series of 634 cases. Zhonghua Wai Ke Za Zhi 2016;54:609-12.

19. Macedo FI, Carvalho EM, Hassan M, et al. Beating heart valve surgery in patients with low left ventricular ejection fraction. J Card Surg 2010;25:267-71.

20. Masroor S, Dance G, Angeli SJ. Beating heart videoassisted mitral valve surgery: a useful technique for highrisk patients. J Card Surg 2009;24:312-4.

21. Lauten A, Ferrari M, Hekmat K, et al. Heterotopic transcatheter tricuspid valve implantation: first-in-man application of a novel approach to tricuspid regurgitation. Eur Heart J 2011;32:1207-13.

Cite this article as: Zhao F, Chen Q, Guo Z, Jiang N. Thoracoscopic assistance in tricuspid valvuloplasty in an unarrested state: a case report and literature review. Ann Transl Med 2020;8(23):1607. doi: 10.21037/atm-20-6995

\section{Zhao et al. Thoracoscopic assistance in tricuspid valvuloplasty}

22. Laule M, Stangl V, Sanad W, et al. Percutaneous transfemoral management of severe secondary tricuspid regurgitation with Edwards Sapien XT bioprosthesis: firstin-man experience. J Am Coll Cardiol 2013;61:1929-31.

23. Rogers T, Ratnayaka K, Sonmez M, et al. Transatrial intrapericardial tricuspid annuloplasty. JACC Cardiovasc Interv 2015;8:483-91.

24. Jeong DS, Kim KH. Tricuspid annuloplasty using the MC3 ring for functional tricuspid regurgitation. Circ J 2010;74:278-83.

25. Rogers JH, Bolling SF. The tricuspid valve: current perspective and evolving management of tricuspid regurgitation. Circulation 2009;119:2718-25.

(English Language Editor: E. Tan) 